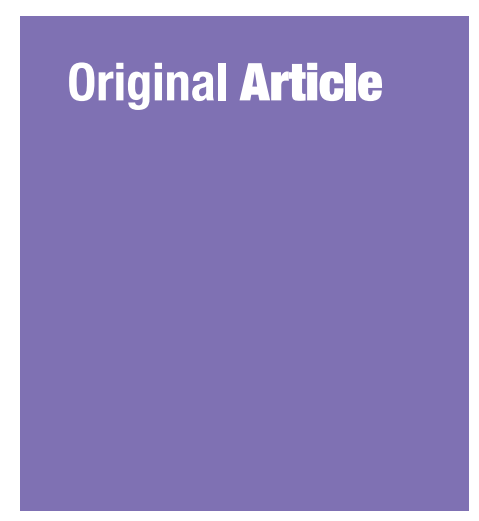

Submitted: 18 Nov 2016 Accepted: 23 Apr 2017 Online: 30 Jun 2017

\section{Inflammatory Biomarkers and Their Value in Predicting Survival and Outcome among Patients with Spontaneous Intracerebral Haemorrhage}

\author{
Senthil Kumar RAJAPATHY, ${ }^{1,2}$, Zamzuri IDRIS ${ }^{1}$, Regunath \\ Kandasamy ${ }^{1}$, Albert Wong Sii Hieng ${ }^{2}$, Jafri Malin Abdullah ${ }^{1,3}$
}

Department of Neurosciences, Universiti Sains Malaysia, Jalan Raja Perempuan Zainab 2, 16150 Kubang Kerian, Kelantan, Malaysia

2 Department of Neurosurgery, Sarawak General Hospital, Jalan Hospital, 93586 Kuching, Sarawak, Malaysia

3 Center for Neuroscience Services and Research, Universiti Sains Malaysia, Jalan Raja Perempuan Zainab 2, 16150 Kubang Kerian, Kelantan, Malaysia

To cite this article: Rajapathy SK, Idris Z, Kandasamy R, Wong ASH, Abdullah JM. Inflammatory biomarkers and their value in predicting survival and outcome among patients with spontaneous intracerebral haemorrhage. Malays J Med Sci. 2017;24(3):51-65. https://doi.org/10.21315/mjms2017.24.3.7

To link to this article: https://doi.org/10.21315/mjms2017.24.3.7

\begin{abstract}
Background: Spontaneous intracerebral haemorrhage (SICH) has emerged as one of the most devastating forms of stroke in recent decades. This disease is noted to carry a 30-day mortality rate of approximately $45 \%$. An increasing number of studies have implicated a complex immune-mediated and inflammation-mediated cascade of responses in the pathophysiology of SICH and the resultant neurologic outcome. Several clinical studies have demonstrated an association between inflammatory markers and outcome in patients with SICH. However, the exact relationship between serum biomarkers and functional outcomes amongst survivors has not been clearly elucidated. This study aimed to evaluate the changes in peripheral leukocyte count (WBC count) and C-reactive protein (CRP) levels in patients with SICH and to correlate these findings with survival and functional outcome.

Methodology: A prospective, descriptive and correlational study was conducted at Sarawak General Hospital (SGH) over the span of two years (April 2013-April 2015). Patients aged between 30 years and 75 years with supratentorial intracerebral bleed secondary to uncontrolled hypertension were recruited in this study. Data pertaining to the demography, clinical and radiological parameters, peripheral WBC count and CRP levels were obtained. Mortality and functional outcomes were determined at 6 months post ictus. Patients were recruited following the fulfilment of exclusion and inclusion criteria, and all obtained data were analysed with the Statistical Package for Social Sciences (SPSS) for Windows version 21.0.

Results: A total of 60 patients with a mean age of 56 years were recruited in this study. We found that approximately 16 patients were less than or equal to 50 years old (26.7\%) and that 44 patients belonged to the older age group of above 50 years $(73.3 \%)$. The Glasgow Coma Scale (GCS) score on admission ranged from 9 to 14/15 with a median value of 11/15. The mean clot volume was $20.1 \mathrm{~cm}^{3}$. The GCS score on admission and clot volume were significantly associated with the Glasgow Outcome Scale (GOS) at 6 months and overall survival $(P<0.05)$. The elevated WBC count and CRP level on admission and at 72 hours post admission were significantly associated with GOS at 6 months and overall survival $(P<0.05)$. Thus, the GCS score, clot volume, WBC count and CRP levels on admission and at 72 hours post admission can be used to predict functional outcome at 6 months and overall survival in patients with SICH.
\end{abstract}

Conclusion: We could conclude via this study that for patients with SICH, the main determinants or predictors of functional outcome at 6 months and overall survival were noted to 
be the GCS score on admission, clot size, WBC count and CRP levels on admission and at 72 hours post admission.

Keywords: intracerebral haemorrhage, white blood cell, leukocyte, C-reactive protein, outcome

\section{Introduction}

Spontaneous intracerebral haemorrhage (SICH) has emerged as one of the most devastating subtypes of stroke. The available contemporary evidence collected from various preclinical studies indicates that SICHinduced inflammation represents a key factor leading to secondary brain damage (1). Epidemiologically, SICH is a leading cause of morbidity and mortality worldwide $(2,3)$. It has been proven to pose worse outcomes than ischemic stroke and has a 30-day mortality of $40 \%-50 \%(4,5)$. Despite increased knowledge of the pathophysiology and advances in the acute treatment of stroke, the outcomes remain poor (6).

Primary SICH accounts for $78 \%$ to $88 \%$ of cases and originates from the spontaneous rupture of small arteries or arterioles damaged by two major causes: hypertensive arteriolosclerosis and amyloid angiopathy $(6,7)$. Early studies have identified hypertension as the most significant modifiable risk factor, occurring in $50 \%-70 \%$ of patients (1). The commonest location of SICH are ganglionic (putamen, caudate nucleus and thalamus), followed by lobar, cerebellar and pontine or brainstem. The mainstay treatment in SICH is merely supportive, the anticipated long-term clinical outcome is generally poor, and the potential extensive burden for primary caretakers is ubiquitous.

There has been more than compelling evidence in recent times to suggest that robust inflammatory mechanisms are triggered in SICH-induced brain injury $(1,8)$. Loftspring et al. (9) found that in animal models, a robust inflammatory response is activated by the entry of blood into the brain parenchyma with a consequent infiltration of peripheral leukocyte or white blood cells (WBC), the activation of microglia and the release of cytokines. These findings were further reciprocated in another study (10). Autopsies conducted in both animals (11) and in human subjects (12) with SICH found leukocytic infiltration usually occurs within the first three days of insult, and further cascadial inflammatory changes in the penumbra of the haemorrhage following $\mathrm{SICH}$ come into play in the natural history of the disease. In the past, very few clinical studies have demonstrated an association between the inflammatory markers and outcomes of SICH in terms of mortality (13, 14). Another inflammatory biomarker that has sparked recent interest in the research of neuroinflammation related to $\mathrm{SICH}$ is C-reactive protein (CRP). These studies have discovered that elevated levels of CRP, an acute-phase reactant induced by IL- 6 , is directly associated with 30-day mortality in SICH patients $(13,14)$.

Several prospective studies in ischemic stroke patients have reported that increased levels of acute inflammatory markers, such as CRP (13-15) and white blood cell (WBC) count (18), were associated with an increased risk of death or disability (16). However, to our knowledge, very few studies have evaluated the functional or long-term outcomes in SICH in relation to WBC and CRP levels.

\section{Materials and Methods}

This study aimed to evaluate the changes in peripheral WBC count and CRP levels on admission and at 72 hours post admission in patients presented with SICH and to correlate these findings with their survival and functional outcome at 6 months.

\section{Sample Selection}

This was a prospective, descriptive and correlational study conducted at Sarawak General Hospital (SGH) over two years (from April 2013 till April 2015). This study was approved by the Malaysian Medical Research and Ethics Committee (MREC) [NMRR ID: 141711-21272 (IIR)]. The sample size for this study was calculated based on the Dupont \& Plummer Power Sample Software version 3.0. The calculated sample size was $60(n)$. The calculated sample size was $60(n)$. We recruited patients aged between 30 and 75 years old presented with supratentorial spontaneous intracerebral haemorrhage (SICH) secondary to uncontrolled hypertension, with a Glasgow Coma Scale (GCS) score on admission between 9 and 14/15 and intracerebral clot size of $10 \mathrm{~cm}^{3}-30 \mathrm{~cm}^{3}$. Patients 
Original Article | Inflammatory biomarkers

with GCS scores of less than 9 on admission, recurrent $\mathrm{SICH}$ or non-hypertensive $\mathrm{SICH}$ were excluded from this study. Other exclusion criteria were patients with infratentorial bleed, intraventricular bleed, brainstem or thalamic bleed, pregnancy, incomplete study duration (minimum 6 months), culture positive infection on admission, or underlying comorbidity (e.g., renal disease, hepatic disease, cancer or chronic systemic autoimmune diseases, such as rheumatoid arthritis or systemic lupus erythematosus). Patients who were treated surgically or had undergone recent surgery or had major trauma $(<4$ weeks prior to $\mathrm{SICH}$ ), patients with a recent ischemic heart disease event, such as myocardial infarction, and patients with acute or chronic infections $(<4$ weeks prior to $\mathrm{SICH}$ ) were also excluded from this study.

\section{Methodology}

We prospectively recruited all patients who fulfilled the inclusion and exclusion criteria, admitted to SGH from April 2013 till April 2015 with a diagnosis of SICH within 24 hours after onset of the stroke. The case subject of SICH was defined as the abrupt onset of spontaneous intracerebral bleeding, which was confirmed by cranial CT scan. Consent was obtained through their family members or legal representatives. These patients underwent full neurological examination; blood tests, which included full blood count (FBC); CRP; renal and liver function tests; coagulation profile; ECG; and chest X-ray. Demographic data, admission GCS scores and haematoma volumes on cranial CTs were documented. The calculation of the haematoma size and volume was based on the Kothari's method using the $\mathrm{A} \times \mathrm{B} \times \mathrm{C} / 2$ formula (17). Blood was collected in ethylenediaminetetraacetic acid (EDTA) containing a tube and plain tube for the assessment of WBC count and CRP level, respectively. The WBC count was determined with an automated haematology analyser (SYSMEX Haemato-analyser), whereas the CRP was determined via a qualitative method using a latex agglutination technique AVITEX-CRP kit (OMEGA Diagnostics). AVITEX-CRP has a detection limit of $5 \mathrm{mg} / \mathrm{L}$ of CRP in the patient's serum, and positive results were obtained at a CRP serum concentration above $5 \mathrm{mg} / \mathrm{L}$. After baseline WBC counts and CRP levels on admission were obtained, the patients were subsequently admitted to the ward for further management. Laboratory personnel were blinded to clinical information, and quality control of assay was maintained.

During the study period, 72 patients with clinical and radiological evidence attributable to SICH were identified. After screening, a total of 60 patients were included in the present study; the remaining 12 patients (16.6\%) were excluded because they did not meet the inclusion criteria. They received all the necessary supportive treatment measures, which included close GCS score monitoring, treatment of hypertension with either oral or intravenous anti-hypertensive medications, initiation of early feeding, and chest and limb physiotherapies. A second set of WBC counts and CRP levels were measured at 72 hours post admission. Patients who encountered clinical deterioration necessitating an emergent surgical procedure were excluded from the study, and the appropriate treatment was delivered to them without any delay. Likewise, patients who developed any signs of infections after hospital admission, such as aspiration pneumonia, hospital-acquired pneumonia, urinary tract infections, hepatitis or thrombophlebitis, were also excluded from the study. Patients who recovered and were clinically stable were discharged, once all the necessary treatment was completed. The Glasgow Outcome Scores (GOS) upon discharge and at 6 months following ictus were recorded.

\section{Statistical analysis}

The clinical data was analysed with the Statistical Package for Social Sciences (SPSS) for Windows version 21.0. The obtained variables were expressed as mean, minimum, maximum, median and standard deviation $(X \pm S D) . A n$ analysis of their interquartile range was also carried out. The correlations of the investigated parameters were analysed with the independent $t$-test, chi-square test, and log rank test (MantelCox test). Correlations between inflammatory marker values and other prognostic factors were also calculated. After examining the distribution, inflammatory marker values were categorised by quartiles for further analyses, and $P$-values of $<0.05$ were considered statistically significant.

\section{Results}

Out of the 60 patients recruited, 36 were males (60\%) and 24 were females (40\%). The mean age was 56.4 years with the range from 32 to 72 years $(S D$ 10.7). The patients were 
divided into two main groups: 50 years old and below; and above 50 years old. There were 16 patients (26.7\%) aged 50 years and below, and 44 patients (73.3\%) aged 50 years and above. Age and gender were not significantly associated with the patients' survival or 6-month functional outcome $(P>0.05)$. Patients recruited under this study were noted to have various compounding co-morbidities. A total of 57 of them (95\%) had underlying hypertension, and the remaining 3 patients $(5 \%)$ had not been previously diagnosed to have hypertension. Eighteen patients (30\%) had underlying diabetes mellitus, and 20 patients (33.3\%) were active smokers, whereas 23 patients (38.3\%) had a history of hypercholesterolemia. Out of the 60 patients, 35 (58.3\%) were on anti-platelets and 5 patients $(8.3 \%)$ had underlying ischemic heart disease. None of these patients experienced any acute cardiovascular-related events that required admission to hospital in the previous 6 months. The systolic blood pressure (SBP) on admission ranged between 145 and $260 \mathrm{mmHg}$, with a mean SBP of $196.5 \pm 26.8 \mathrm{mmHg}$. Diastolic blood pressure (DBP), however, ranged between 79 and $140 \mathrm{mmHg}$, with a mean DBP of $110.9 \pm$ 15.1. The mean arterial pressure (MAP) ranged from 107 to 175 with a mean MAP of 139.5 (SD \pm 17.3)

The mean GCS score was 11/15 with the range of 9 to 14 . The median GCS score was 11 with an Interquartile Range of 10 to 12 (SD \pm 1.6). Majority of patients presented with GCS score of 9 to 11 with a total of 13 patients (21.7\%) in each GCS score group of 9, 10 and 11. There were only 5 patients $(8.3 \%)$ in the group with GCS score of 14 . By using independent $t$-tests, the GCS score was noted to be significantly associated with 6-month functional outcome based on the GOS and overall mortality. CT images of the brain revealed that out of the 60 recruited patients, the majority presented with bleed over the right supratentorial region (32 patients), making up about $53.3 \%$ of the total number of patients. The remaining 28 (46.7\%) had SICH over the left supratentorial region. About 54 patients (90\%) presented with bleed in the ganglionic region (caudate nucleus, putamen and globus pallidus), and the remaining 6 patients (10\%) presented with bleed in the cortical lobar region. The volume of the clot or intracerebral bleed (ICB) ranged from a minimum volume of $10.0 \mathrm{~cm}^{3}$ to a maximum of $30.0 \mathrm{~cm}^{3}$, with a mean clot size of $20.1 \mathrm{~cm}^{3}(\mathrm{SD} \pm$ 6.9). The interquartile range (IQR) was found to be between 14.5 and $26.6 \mathrm{~cm}^{3}$.
When the patients were analysed according to functional outcome based on GOS at 6 months, the size of clot was noted to be one of the dictating factors. Patients were divided into 2 groups, one with a poor GOS score of 2 to 3 and another group with a good GOS score of 4 to 5 . A total of 20 patients (33.3\%) belonged to the better GOS group, and 27 (45\%) of patients belonged to the poor GOS group. Thirteen patients (21.7\%) succumbed to the disease. When tested with independent $t$-tests, morbidity and overall mortality or survival was found to be significantly associated with the volume of clot at presentation $(P<0.05)$ (Table 1$)$.

As illustrated in Table 1, the volume of clot and GCS score on admission were found to significantly contribute to the 6-month functional outcome based on GOS when examined with an independent $t$-test $(P<0.05)$. An elevated WBC count on admission and at 72 hours also predicted a worse outcome or a poor GOS at 6 months $(P<0.05)$ when tested with an independent $t$-test. Likewise, an elevated CRP level (> $5 \mathrm{mg} / \mathrm{L}$ ) on admission and at 72 hours following admission was also related to poorer functional outcome at 6 months. Patients with elevations of both WBC counts and CRP levels on admission and at 72 hours post admission tended to have worse GOS in 6 months (GOS 2 to 3). The WBC count was also found to be strongly correlated with haematoma volume at admission. Patients with a larger clot size tend to have a more profoundly elevated WBC count on admission and at 72 hours post admission.

The volume of clot, GCS score on admission, and WBC count on admission and at 72 hours were also found to predict 6-month mortality. The elevated WBC counts increased the mortality rate among these patients $(P<0.05)$ when measured with independent $t$-tests. However, no correlation was found between an elevated CRP level (> $5 \mathrm{mg} / \mathrm{L}$ ) on admission and at 72 hours following admission and mortality at 6 months $(P>0.05)$. As shown in Table 1 , the mortality of patients with SICH was significantly associated with the volume of clot, GCS score on admission and WBC count on admission as well as at 72 hours following admission.

The baseline WBC count on admission ranged from 4.7 to $34.8 \times 10^{3} / \mathrm{L}$. The mean WBC count was $16.1 \times 10^{3} / \mathrm{L}$, and the median was $15.3 \times 10^{3} / \mathrm{L}(\mathrm{SD} \pm 8.0)$. The interquartile $25^{\text {th }}$ and $75^{\text {th }}$ percentiles were 9.4 and $20.2 \times$ $10^{3} / \mathrm{L}$, respectively. The $\mathrm{WBC}$ count at 72 hours post admission ranged from 5.8 to $28.0 \times 10^{3} / \mathrm{L}$. The mean WBC count was $15.063 \times 10^{3} / \mathrm{L}$, and 
the median was $13.8 \times 10^{3} / \mathrm{L}(\mathrm{SD} \pm 6.0)$. The interquartile range was between 10.6 and $19.4 \times$ $10^{3} / \mathrm{L}$. The WBC count was further divided into 4 quartiles (Q1, Q2, Q3 and Q4) to facilitate the feasibility of drawing any conclusions (Table 2).

This study also found that a large number of patients had elevated CRP level (> $5 \mathrm{mg} / \mathrm{L}$ ) on admission and at 72 hours post admission. A total of 48 patients (80\%) presented with elevated CRP levels on admission, and 40 of them (66.7\%) showed continued elevated CRP levels at 72 hours post admission. The drop in the number at 72 hours post admission was due to the death of 8 patients before the CRP level assessment at 72 hours following admission (Table 3).

It was observed that patients within the 3rd and 4th WBC count quartiles on admission tended to have worse GOS at 6 months, as depicted in Table 4. Patients within the lower 1st and 2nd WBC count quartiles on admission tended to have a better functional outcome at 6 months $(P<0.05)$. A good functional outcome in this context refers to patients with GOS 4 and 5 .

Out of the 47 surviving patients (excluding the 13 patients who succumbed to the disease eventually), 27 patients (57.4\%) exhibited poor functional outcome (GOS $2-3$ ), and 20 patients (42.6\%) showed good functional outcome (GOS 4-5)(Table 5). Figure 1 shows that 6-month functionality was good for patients within the 1st and 2nd quartiles of the WBC count. Patients with the higher WBC counts within the 3rd and 4th quartiles showed poor functionality at 6 months. When the findings above were analysed with a Mantel-Cox test of equality of survival (degree of freedom, $d f=3$ ), there was a significant difference in the functionality rate at 6 months between the different quartiles of the WBC count on admission ( $P$-value < 0.05)(Figure 2). Therefore, the WBC count on admission was significantly associated with functionality at 6 months.

Table 6-Table 8 showed that when the WBC count and CRP levels were evaluated and divided specifically into quartiles, the temporal relationship of the elevated WBC count and CRP to the general 6-month outcome in patients could be determined. In general, a low WBC count and CRP level on admission were significantly associated with good functional outcome (GOS 4 and 5), and an elevated WBC count and CRP level were associated with poor functional outcome (GOS 2 and 3) ( $P$-value < 0.05).
The relationship of elevated $\mathrm{WBC}$ count at 72 hours post admission to poor 6-month functionality was also examined. (Table 9-Table 11) The data revealed that poorer functional outcomes were observed in patients within the group with elevated WBC counts (in the 3 rd and 4th quartiles). An analysis of the data also found a significant difference in 6-month functionality or GOS among the different quartiles of the WBC count at 72 hours $(P$-value $<0.05)$. The MantelCox test of equality of survival distributions for the different quartiles of WBC and CRP at 72 hours post admission revealed a significant $P$-value of $<0.05$ (chi-square 24.398 with a degree of freedom of 5). Therefore, it could be concluded that there is a significant difference in the functionality rate among different quartiles of the WBC count and CRP at 72 hours ( $P$-value $<$ 0.05). As demonstrated in Table 12 and 13, high WBC counts and CRP levels on admission are significantly related to increased mortality $(P$-value $<0.05)$

\section{Discussion}

Neuro-inflammatory mechanisms and cascades are exceedingly recognised as substantially important players in the pathophysiology of secondary brain damage after SICH. The role of inflammation in the pathophysiology of SICH could not be emphasised enough (31). SICH is considered one of the critical forms of aseptic inflammation mounted as a response to damage incurred by intracerebral haemorrhage. More and more studies have provided tangible evidence that leukocytes play a substantial role in the propagation of disease and the consequent deterioration of patients with SICH. Patients presented with higher WBC counts were found to have larger intracerebral clots. Studies have also proved that elevated WBC counts predict early neurologic deterioration, worse outcome and mortality after $\mathrm{SICH}(4,17)$. It can be postulated that if a marked leukocytic response is seen in a patient with acute $\mathrm{SICH}$, one would expect that the mechanism that is at work would more likely reflect a systemic stress-induced response rather than a local inflammatory response in the brain. However, such a robust and more systemic stress-induced mechanism of leukocytosis remains hypothetical. In the acute phase of stroke, inflammation contributes to brain damage triggered by ischemia $(7,8)$. The inflammatory cascade is triggered by an 
increasing concentration of local cytokines, adhesion molecules, acute phase proteins, macrophages and leukocytes, and the strength of this response is noted to be directly related to early and late clinical outcomes (5).

The role of CRP in the acute phase response to stroke has also been extensively investigated $(7,8)$. A marked elevation in CRP levels has been routinely found after an episode of ischemic or haemorrhagic stroke and is associated with a worsened outcome and the increased in risk of developing other cerebrovascular issues, such as further ischemic stroke, and cardiovascular events, such as myocardial infarction, in stroke patients (9).

The present study corroborates the various findings and postulated theories found in previous studies, as mentioned earlier. Often following SICH, an inflammatory response is mounted at the site of an intracerebral haematoma in human subjects (21). Evidence that is more than compelling suggests that the inflammatory response may directly contribute to SICH-related injury (31). Neurologic deterioration in ICH is most likely to occur within the first 48 hours after onset (17). WBCs can promote inflammatory tissue injury indirectly by stimulating macrophages to release pro-inflammatory mediators and via the effects of oxygen-free radicals and complement system activation (15). The data collected in this study illustrate that patients with higher WBC counts had greater haematoma volume at admission. Hence, it can be put forth that WBC count may play a role as a potential biomarker of a heightened inflammatory response and may be a direct contributor to haematoma growth and brain injury sustained due to SICH. This haematoma growth may be attributed to early neurologic deterioration in patients. A previous study by Liera et al. (17) showed that higher WBC counts were associated with early neurologic deterioration, but were not independently associated with functional outcomes as measured by the Modified Rankin Scale at 30 days. Studies have also demonstrated that although a higher WBC count was observed with increased mortality in SICH patients, this did not increase the risk of death independently of other indicators of SICH severity (17). In the present study, we observed convincing relationships among baseline inflammatory markers (WBC count and CRP level) and mortality. These observations are in line with previous studies conducted involving WBC count $(11,17)$ and also with studies of other inflammatory markers $(6,7)$.

The degree of WBC elevation also reflects the severity of SICH. This theory was supported by our data, in which we were able to demonstrate correlations between elevated WBC count and both haematoma volume at admission and SICH severity. Therefore, it could be postulated that elevated WBC count on admission and at 72 hours following admission greatly dictate and determine the overall 6-month functionality as assessed by GOS scores and is also related to 6-month survival. We proved that WBC could serve as an important early biomarker in assessing SICH severity and outcome. WBC count is an important and inexpensive tool available in all hospitals locally and is universally assayed in all patients with $\mathrm{SICH}$. The value for the lowest quartile of WBC count in our study was $10.6 \times 10^{3} / \mathrm{L}$, which is also close to the upper limit of normal for many laboratories. Studies involving vascularspecific anti-inflammatory and antileukocyte therapies are seeking to develop an alternative or adjunctive therapeutic approach to SICH (31); an elevated WBC count may help identify patients who would benefit the most from these interventions.

To our knowledge, this is the first study conducted locally that evaluates the direct relationship between both CRP and WBC counts and 6-month functionality and mortality in SICH patients. WBC count and CRP level at admission and at 72 hours following admission clearly demonstrated a strong association $(P<$ 0.05) with 6-month functionality. This is in line with previously made observations (33). The relationship between the acute phase-reactant CRP inflammatory marker status and WBC count on admission also has a direct causal effect on 6-month mortality or death. The patients in our study presenting with elevated WBC count with a positive CRP value succumbed to the disease before 6 months. It is conceivable that an increased CRP concentration after SICH onset is due to inflammation related to the pathophysiology of intracerebral haematoma growth and might reflect the extent of the haematoma volume and not to a pre-existent subclinical inflammatory status. Experimental data has demonstrated that the acute phase response to intracerebral haematoma occurs later when compared to ischemic stroke (22). Gong et al. (12) noted that interleukin-6 (IL-6), a pro-inflammatory cytokine that regulates the level of CRP (20), was observed in the blood, 
Original Article | Inflammatory biomarkers

Table 1. Baseline characteristics and potential baseline factors associated with 6-month functional outcome and mortality

\begin{tabular}{|c|c|c|c|c|c|c|c|}
\hline Characteristics & $\begin{array}{c}\text { Total } \\
\text { Cohort } \\
(n=60)\end{array}$ & $\begin{array}{c}\text { Better } \\
(\text { GOS 4-5) } \\
(n=20) \\
\end{array}$ & $\begin{array}{c}\text { Worse } \\
(\text { GOS 2-3) } \\
(n=27) \\
\end{array}$ & $P$-value & $\begin{array}{c}\text { Alive } \\
(n=47)\end{array}$ & $\begin{array}{c}\text { Death } \\
(n=13)\end{array}$ & $P$-value \\
\hline Age, years (SD) & $\begin{array}{l}56.35 \\
(10.66)\end{array}$ & $\begin{array}{l}54.5 \\
(10.21)\end{array}$ & $\begin{array}{l}57.41 \\
(9.33)\end{array}$ & 0.316 & $\begin{array}{l}56.17 \\
(9.71)\end{array}$ & $57.00(14.02)$ & 0.844 \\
\hline Gender, male n (\%) & $36(60 \%$ & $15(75 \%)$ & $12(44.4 \%)$ & 0.072 & $27(57.4 \%)$ & $9(69.2 \%)$ & 0.654 \\
\hline Clot, $\mathrm{cm}_{3}$ (SD) & $\begin{array}{l}20.11 \\
(6.78)\end{array}$ & $\begin{array}{l}13.63 \\
(3.19)\end{array}$ & $\begin{array}{l}22.03 \\
(6.03)\end{array}$ & $0.000^{*}$ & $\begin{array}{l}18.46 \\
(6.51)\end{array}$ & $26.01(3.78)$ & ${ }^{*} 0.000$ \\
\hline GCS, median (IQR) & $11(10-12)$ & $\begin{array}{l}13(12- \\
13 \cdot 75)\end{array}$ & $\begin{array}{l}10.00 \\
(10-11)\end{array}$ & $0.000^{*}$ & $11(10-13)$ & $9(9-9.5)$ & ${ }^{*} 0.000$ \\
\hline $\begin{array}{l}\text { WBC on admission, } \\
\text { x } 103 \text { median (IQR) }\end{array}$ & $\begin{array}{l}15 \cdot 3 \\
\left(9.45^{-}\right. \\
20.18)\end{array}$ & $\begin{array}{l}9.55 \\
\left(5 \cdot 93^{-}\right. \\
12.3)\end{array}$ & $\begin{array}{l}17.6 \\
(11.3-23.7)\end{array}$ & $0.000^{*}$ & $\begin{array}{l}12.5\left(8.4^{-}\right. \\
18.4)\end{array}$ & $\begin{array}{l}23.9(16.6- \\
29.5)\end{array}$ & ${ }^{*} 0.000$ \\
\hline $\begin{array}{l}\text { WBC at } 72 \text { hours, } x \\
103 \text { median (IQR) }\end{array}$ & $\begin{array}{l}13.8 \\
(10.6- \\
19.4) \\
n=51\end{array}$ & $\begin{array}{l}10.25\left(7.5^{-}\right. \\
13.7)\end{array}$ & $\begin{array}{l}17.8 \\
(13-22.4)\end{array}$ & $0.000^{*}$ & $\begin{array}{l}13.8(9.9- \\
18.6) \\
n=47\end{array}$ & $\begin{array}{l}19.15(17.4- \\
20.08) \\
n=4\end{array}$ & ${ }^{*} 0.002$ \\
\hline $\begin{array}{l}\text { CRP-positive on } \\
\text { admission, } \\
>5 \mathrm{mg} / \mathrm{L}, \mathrm{n}(\%)\end{array}$ & $48(80 \%)$ & $8(40 \%)$ & $27(100 \%)$ & $0.000^{*}$ & $35(74.5 \%)$ & $13(100 \%)$ & 0.100 \\
\hline $\begin{array}{l}\text { CRP-positive at } 72 \\
\text { hours, } \\
>5 \mathrm{mg} / \mathrm{L}, \mathrm{n}(\%)\end{array}$ & $\begin{array}{l}40 \\
(76.9 \%) \\
n=52\end{array}$ & $8(40 \%)$ & $27(100 \%)$ & $0.000^{*}$ & $\begin{array}{l}35(74.5 \%) \\
n=47\end{array}$ & $\begin{array}{l}5(100 \%) \\
n=5\end{array}$ & 0.465 \\
\hline
\end{tabular}

Table 2. Quartile values for WBC count on admission and at 72 hours of admission

\begin{tabular}{lcccc} 
& Q1 & Q2 & Q3 & Q4 \\
WBC count (X103) on admission & 9.450 & 15.300 & 20.175 & 34.8 \\
WBC count (X103) at 72 hours of admission & 10.600 & 13.800 & 19.400 & 28.00 \\
\hline
\end{tabular}

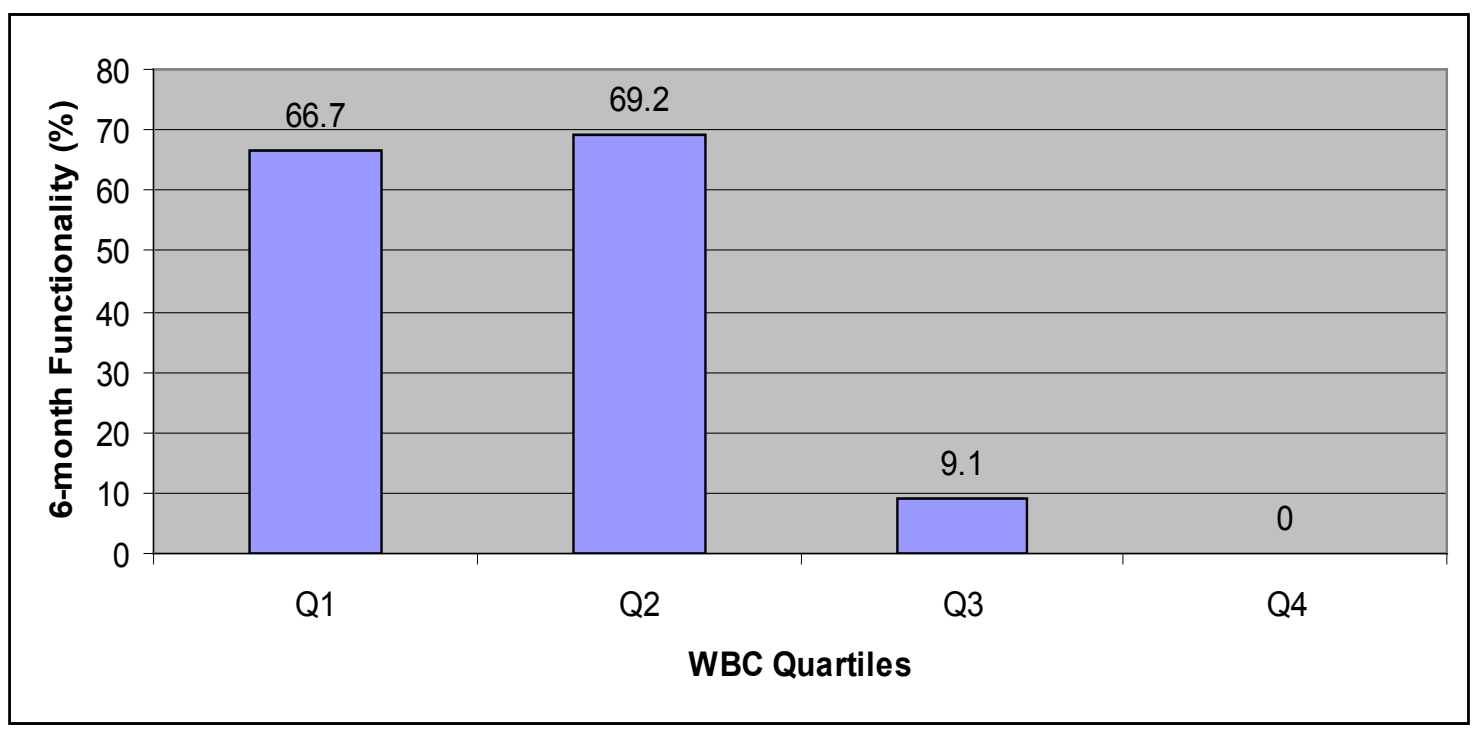

Figure 1. Six-month functionality according to WBC count quartiles on admission $(n=47)$ 


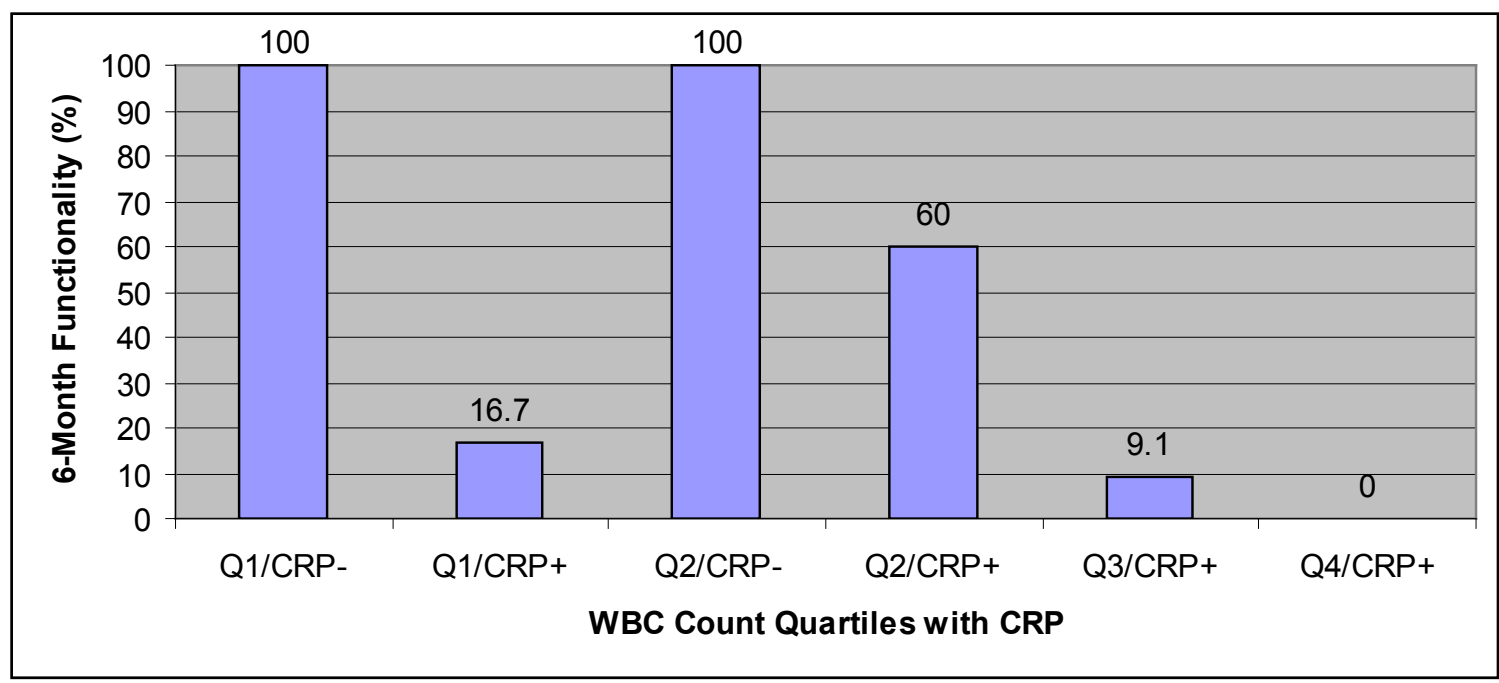

Figure 2. Six-month functionality according to WBC count quartiles with CRP on admission $(n=47)$

Table 3. Patients with elevated CRP levels on admission and at 72 hours of admission

\begin{tabular}{|c|c|c|}
\hline CRP > $5 \mathrm{mg} / \mathrm{L}$ on Admission & Frequency & Percentage (\%) \\
\hline Positive & 48 & 80.0 \\
\hline Negative & 12 & 20.0 \\
\hline Total & 60 & 100.0 \\
\hline $\begin{array}{l}\text { CRP }>5 \mathrm{mg} / \mathrm{L} \text { at } 72 \text { hours of } \\
\text { admission }\end{array}$ & Frequency & Percentage (\%) \\
\hline Positive & 40 & 66.7 \\
\hline Negative & 12 & 20.0 \\
\hline Total & 52 & 86.7 \\
\hline $\begin{array}{l}\text { Missing } \\
\text { (patient died before } 72 \text { hours) }\end{array}$ & 8 & 13.3 \\
\hline Total & 60 & 100.0 \\
\hline
\end{tabular}

Table 4. Functionality (\%) of patients at 6 months based on the quartiles of WBC

\begin{tabular}{lccc} 
WBC Quartiles on Admission & Total $(N)$ & $N$ of Functionality & Functionality (\%) \\
1 & 15 & 10 & 66.67 \\
2 & 13 & 9 & 69.23 \\
3 & 11 & 1 & 9.09 \\
4 & 8 & 0 & 0.00 \\
$P$-value & & & 0.000 \\
\hline
\end{tabular}


Original Article | Inflammatory biomarkers

Table 5. Relationship between GOS and WBC quartiles on admission

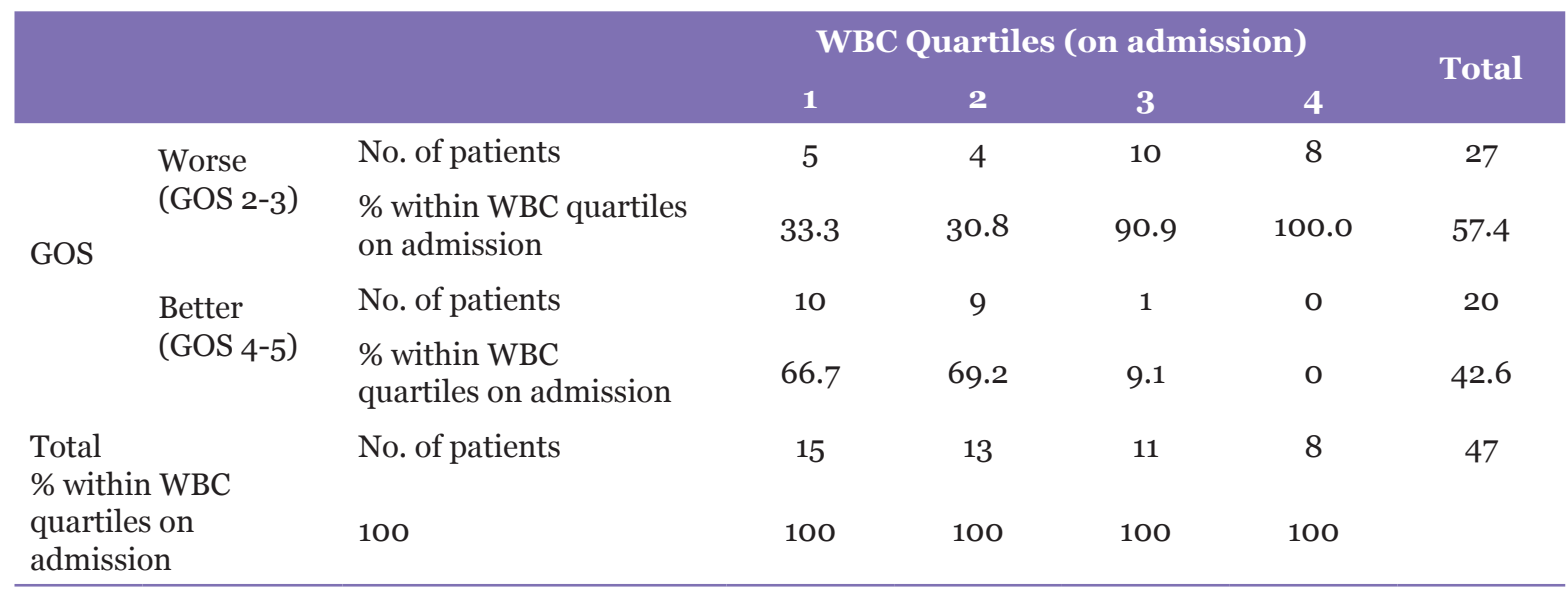

Table 6. GOS versus WBC quartiles with CRP reactivity

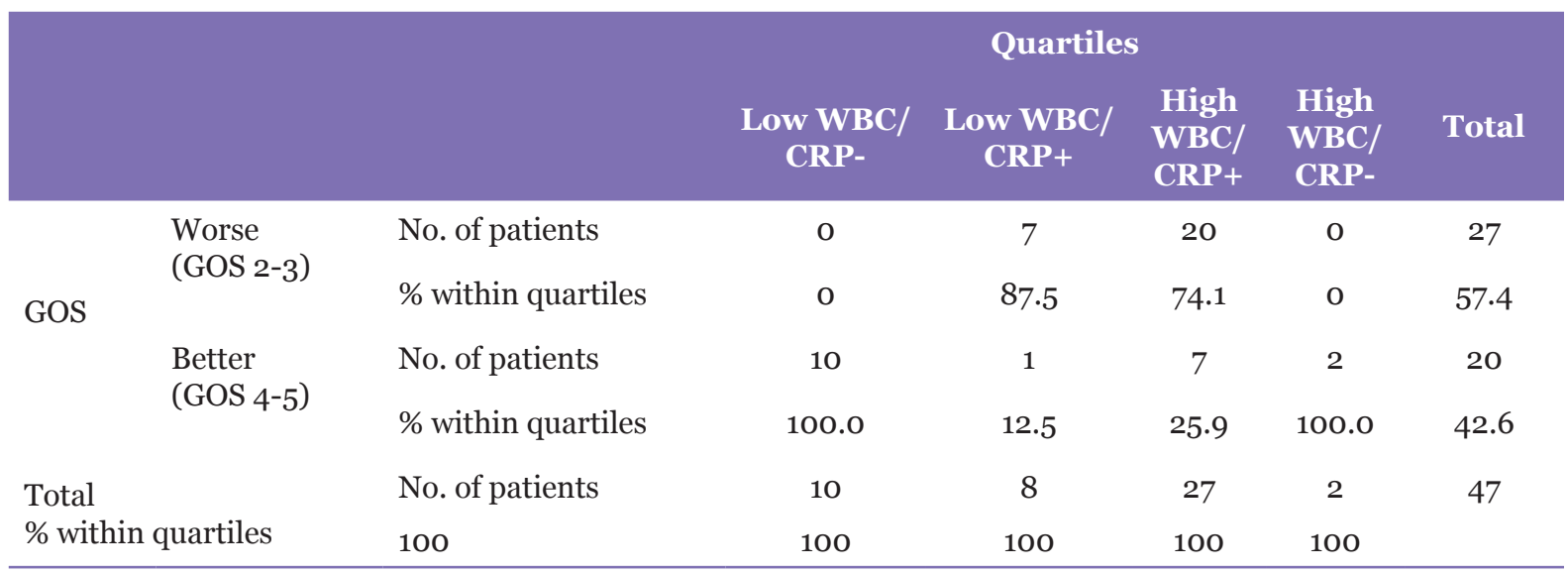

Table 7. GOS according to quartiles of WBC and CRP

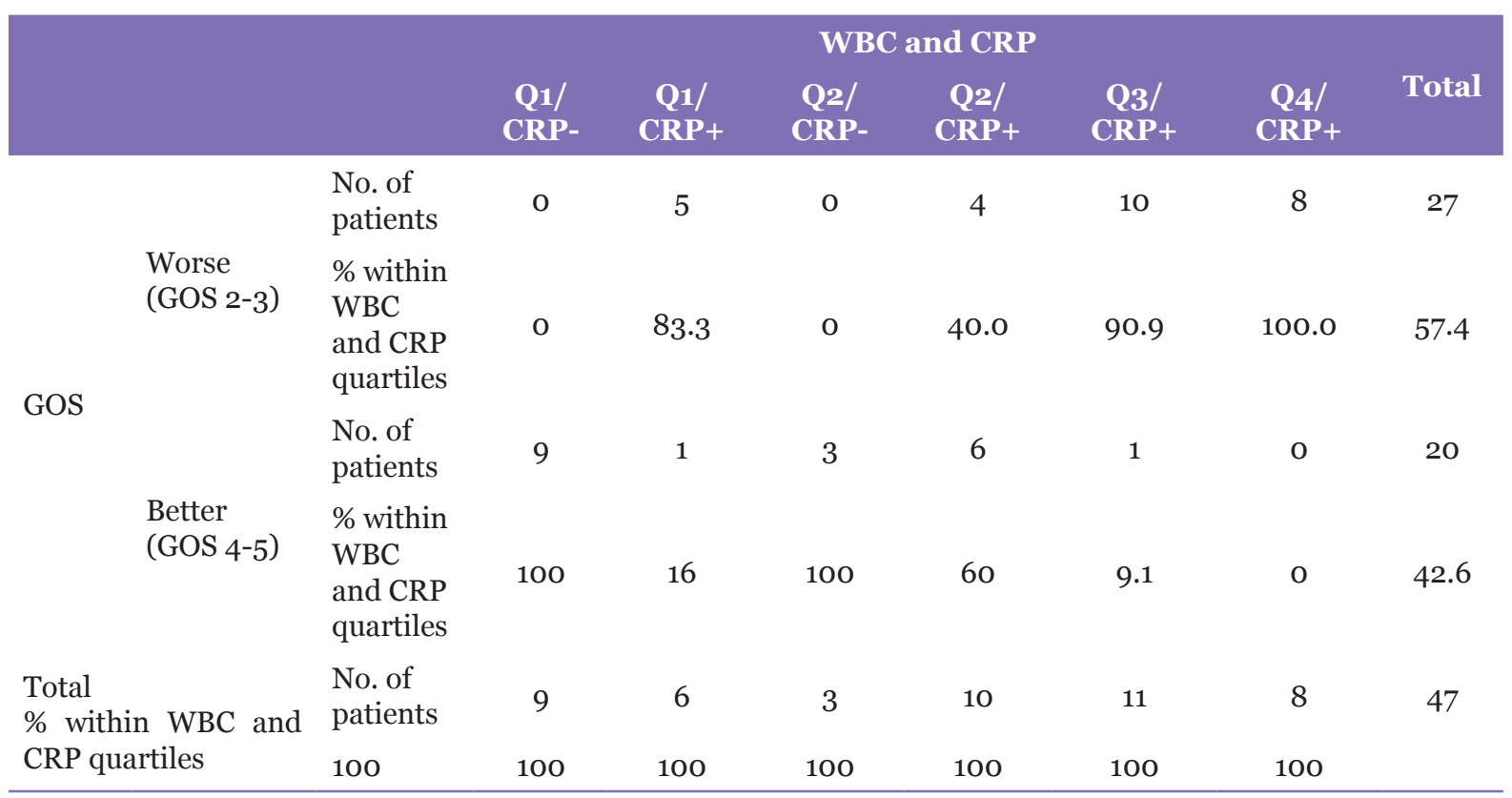


Table 8. Functionality based on WBC quartiles and CRP

\begin{tabular}{lccc} 
WBC Quartiles/CRP & Total $(N)$ & $N$ of Functionality & Functionality (\%) \\
Q1/CRP- & 9 & 9 & 100.00 \\
Q1/CRP+ & 6 & 1 & 16.67 \\
Q2/CRP- & 3 & 3 & 100.00 \\
Q2/CRP+ & 10 & 6 & 60.00 \\
Q3/CRP+ & 11 & 1 & 9.09 \\
Q4/CRP+ & 8 & 0 & 0.00 \\
$P$-value & & & 0.000 \\
\hline
\end{tabular}

Table 9. Relationship of GOS with different WBC quartiles at 72 hours of admission

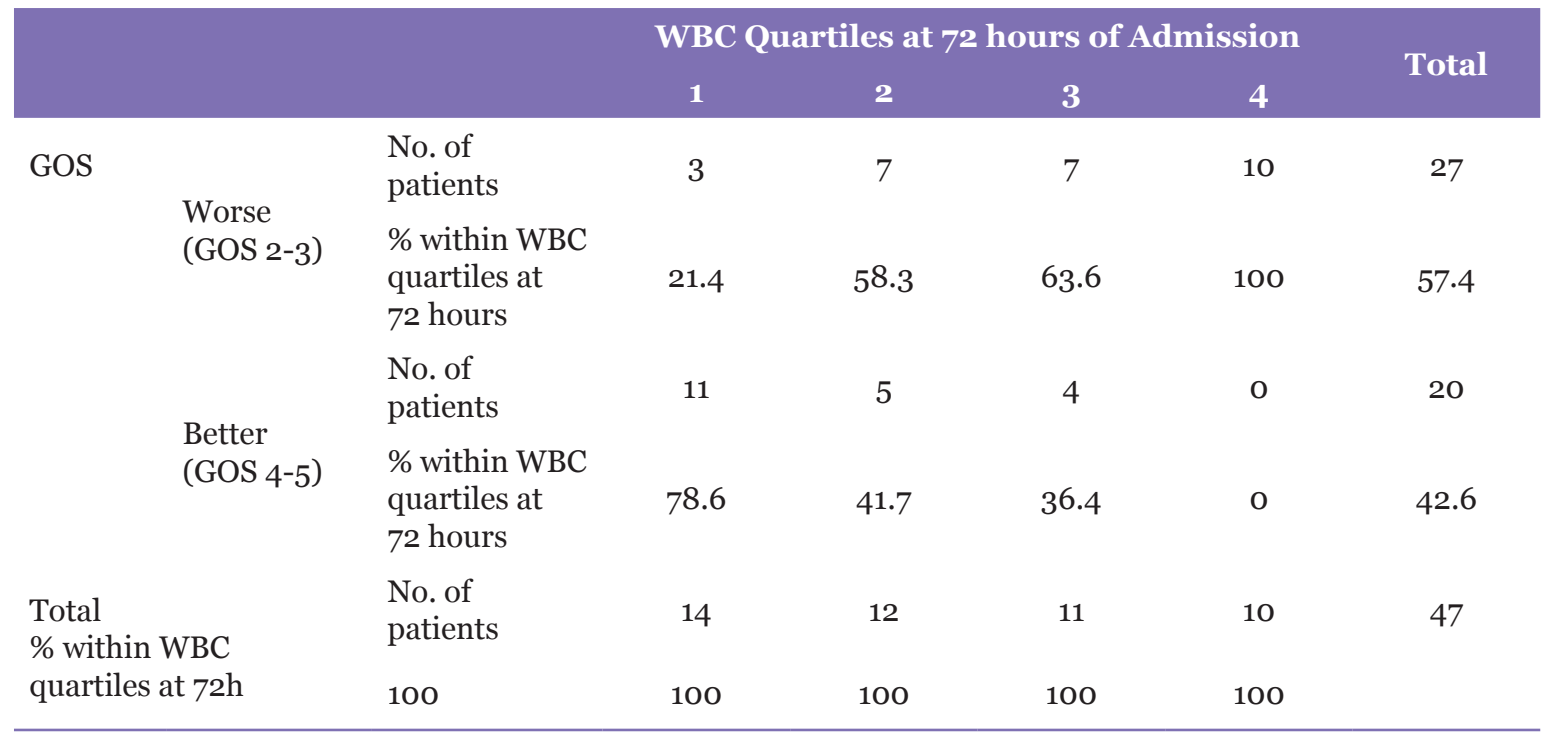

Table 10. Functionality based on WBC quartiles at 72 hours of admission

\begin{tabular}{cccc}
$\begin{array}{c}\text { WBC Quartiles at } 72 \text { hours of } \\
\text { Admission }\end{array}$ & Total $(N)$ & $N$ of Functionality & Functionality (\%) \\
1 & 14 & 11 & 78.6 \\
3 & 12 & 5 & 41.7 \\
4 & 11 & 4 & 36.4 \\
$P$-value & 10 & 0 & 0.0 \\
\hline
\end{tabular}

Table 11. Relationship between WBC quartiles and CRP at 72 hours of admission 
Original Article | Inflammatory biomarkers

$\begin{array}{cccc}\text { WBC and CRP Quartiles at 72 hours } & \text { Total }(N) & N \text { of Functionality } & \text { Functionality (\%) } \\ \text { Q1/CRP- } & 10 & 10 & 100 \\ \text { Q1/CRP+ } & 4 & 1 & 25 \\ \text { Q2/CRP- } & 2 & 2 & 100 \\ \text { Q2/CRP+ } & 10 & 3 & 30 \\ \text { Q3/CRP+ } & 11 & 4 & 36.4 \\ \text { Q4/CRP+ } & 10 & 0 & 0 \\ P \text {-value } & & & 0.000\end{array}$

Table 12. Relationship of WBC quartiles on admission to mortality rate

\begin{tabular}{cccc} 
WBC Quartiles on Admission & Total $(N)$ & $N$ of Death & Mortality Rate (\%) \\
1 & 15 & 0 & 0.00 \\
2 & 15 & 2 & 13.33 \\
4 & 15 & 4 & 26.67 \\
$P$-value & 15 & 7 & 46.67 \\
\hline
\end{tabular}

Table 13. Relationship of WBC quartiles with CRP on admission to mortality rate

\begin{tabular}{cccc}
$\begin{array}{c}\text { WBC and CRP } \\
\text { Quartiles on Admission }\end{array}$ & Total $(N)$ & $N$ of Death & $\begin{array}{c}\text { Mortality } \\
\text { Rate (\%) }\end{array}$ \\
Q1/CRP- & 9 & 0 & 0.00 \\
Q1/CRP+ & 6 & 0 & 0.00 \\
Q2/CRP- & 3 & 0 & 0.00 \\
Q2/CRP+ & 12 & 2 & 16.67 \\
Q3/CRP+ & 15 & 4 & 26.67 \\
Q4/CRP+ & 15 & 7 & 46.67 \\
$P$-value & & & 0.037 \\
\hline
\end{tabular}


cerebrospinal fluid or brain extracts at 1 hour after experimental SICH. Other data obtained regarding the temporal course of CRP in humans showed a more delayed response $(25,31)$. These findings may suggest that the prediction of patients who are more predisposed to a robust and heightened inflammatory response and who will thus deteriorate could only be determined by serial CRP measurements after the onset of symptoms. However, in this study, a clear significant relationship between CRP levels obtained early at the time of admission and 6-month survival and functionality could be established.

We also found that patients with an elevated WBC and CRP concentration on admission had a relatively higher risk of dying at 6 months. Patients in the 3rd WBC quartile with positive CRP had a mortality rate of $26.67 \%$, and patients in the 4th WBC quartile with positive CRP had a mortality rate of $46.67 \%$. Patients belonging to the 1st and 2nd WBC quartiles with positive CRP survived overall. In patients with a low WBC count, 6-month mortality was related to the baseline CRP concentration. Thus, the combined use of baseline WBC count and CRP concentration can be used to stratify and predict mortality risk. We did not find a causal relationship between both WBC and CRP level at 72 hours on admission with 6-month survival or mortality. This was probably due to the small sample size in this study.

With regards to the haematoma volume, we found that it was a significant predictor of 6-month functionality and survival $(P<0.05)$. A larger haematoma volume causes midline shift and is associated with a 7.5 fold risk of mortality. Haematoma volume has been previously attributed to mortality in many published studies $(13,19)$, and it has been found that intracerebral bleed volume is a significant independent predictor of 30-day mortality.

We also found that functional outcomes at 6 months are significantly associated with GCS scores on admission. This finding is in accordance with previously conducted studies which have concluded that GCS is a consistent predictor of outcome in patients with SICH $(14,24)$. This study also observed no strong correlation between age and gender of patients with overall 6-month functionality or mortality. This is perhaps due to the relatively small sample size. Gender effect on the SICH outcome was previously studied by Smaljlovic et al (26), and they concluded that gender has no prognosticating value in SICH. The relationship between age and SICH outcome was also previously investigated (10). The researchers found that age is an independent predictor of 30-day mortality and that age greater than 60 years old is a strong predictor of mortality and functional outcome.

The present study design has several limitations. As an observational study, it could only identify associations and not ultimate causality. We did not collect detailed information on the WBC differential and absolute quantitative CRP value, which may be important. An association and relationship between the differential WBC count and CRP level to the 6-month functionality and mortality could probably be demonstrated more objectively if a quantitative analysis were undertaken. Perhaps we could have demonstrated a strong association of a particular WBC lineage, such as polymorphonuclear leukocytes and monocytes, to the overall clinical course and outcomes in patients with SICH. A quantitative method and analysis, however, requires additional fund support, which was not economically feasible. The CRP level estimated in this study was merely qualitative, and perhaps a quantitative measurement could provide more compelling evidence of causality and relationship.

This study also did not examine the relationship of other known inflammatory markers attributed in the pathophysiology of the neuro-inflammation that follows SICH, such as interleukin 1,6, tumour necrosis factor (TNF), and others. A venture of that nature would require adequate funds and personnel to make it a reality and was beyond the purview of this research.

\section{Conclusion}

Based on this study, we concluded that 6-month functional outcome, mortality or survival in patients with SICH were significantly associated with both the volume of intracerebral bleed and GCS score on admission. We also found that WBC counts and CRP levels on admission and at 72 hours post admission were significantly associated with functional outcome at 6 months. WBC counts and CRP levels on admission were significantly associated with 6-month mortality. The assay of these bio-inflammatory markers could be used to predict mortality and functionality of patients presenting with $\mathrm{SICH}$ and therefore enable prompt counselling and insight to family 
Original Article | Inflammatory biomarkers

members regarding the prognosis of patients. This study also sheds lights on novel, potentially revolutionary treatments of SICH, such as antiflammatory and neuro-modulation approaches to treat haemorrhagic stroke.

\section{Acknowledgement}

This manuscript represents not only my work at the keyboard but also an important milestone in the completion of my masters' degree training in the field of neurosurgery. Prima facie, I would like to express my sincere gratitude to all those who have contributed directly or indirectly in my quest to complete this dissertation.

First and foremost, I would like to express my utmost gratitude and appreciation to my dissertation supervisors Assoc Prof Dr Zamzuri Idris (Head of Department of Neurosciences, Hospital Universiti Sains Malaysia), Mr Albert Wong Sii Hieng (Head of Department and Senior Consultant Neurosurgeon, Hospital Umum Sarawak) and Mr Donald Liew Ngai San (Consultant Neurosurgeon, Hospital Umum Sarawak). I thank them for their continuous guidance, patience, motivation, and immense knowledge. Their support and encouraging and timely advises has helped me in completing this thesis.

I also place on record, my sincere thank you to Mr Regunath (Consultant Neurosurgeon, Department of Neurosciences, Hospital Universiti Sains Malaysia), Assoc Prof Dato' Abdul Rahman Izani Ghani (Consultant Neurosurgeon, Department of Neurosciences, Hospital Universiti Sains Malaysia), and also to Mr Lim Swee San (Neurosurgeon, Hospital Umum Sarawak). Not forgetting to thank at this point is the founding father of the Neurosurgical Residency Training Programme at Universiti Sains Malaysia-Professor Dato' Dr Jafri Malin Abdullah. I am extremely thankful and indebted to them for sharing their expertise, and for the sincere and invaluable guidance and encouragement extended to me. It is wholeheartedly expressed that their advices for my research proved to be a landmark effort towards the success of my dissertation

I would also like to take this opportunity to thank my colleagues and the nursing staff in both of my training centres-namely Sarawak General Hospital and Hospital Universiti Sains Malaysia for their undivided and relentless support and help with data collection and compilation. Last but not the least, I would like to accord my sincere gratitude to my parents and family for their unwavering encouragement and support throughout my endeavours. I would like to thank all those whose assistance proved to be a milestone in the accomplishment of my end goal and also place on record, my sense of gratitude to one and all, who directly or indirectly, have lent their hand in this venture of mine.

\section{Conflict of Interest}

The authors declare that there is no conflict of interest related to this study.

\section{Authors' Contributions}

Conception and design: SKR

Analysis and interpretation of the data: SKR

Drafting of the article: SKR

Critical revision of the article for important intellectual content: ZI, RK, AWSH, JMA

Final approval of the article: JMA

Provision of study materials or patients: AWSH

\section{Correspondence}

Dr Senthil Kumar Rajapathy

MBBS (Manipal), MS (Neurosurgery) (USM)

Department of Neurosciences,

Universiti Sains Malaysia,

Jalan Raja Perempuan Zainab 2,

16150 Kubang Kerian,

Kelantan, Malaysia.

Tel: +6012 4757067

E-mail: senthilkraj8o@gmail.com

\section{References}

1. Aronowski J, Hall CE: New horizons for primary intracerebral hemorrhage treatment: experience from preclinical studies. Neurol Res. 2005;27:268-279. https://doi.org/10.1179/01616 $4105 \mathrm{X} 25225$

2. Broderick JP, Adams HP Jr, Barsan W, Feinberg W, Feldmann E, et al. Guidelines for the management of spontaneous intracerebral hemorrhage: a statement for healthcare professionals from a special writing group of the Stroke Council, American Heart Association. Stroke. 1999;30:905-915. https://doi.org/10. 1161/01.STR.30.4.905 
3. Broderick JP, Brott T, Tomsick T, Miller R, Huster. Intracerebral hemorrhage more than twice as common as subarachnoid hemorrhage. $J$ Neurosurg. 1993;78:188-191. https://doi. org/10.3171/jns.1993.78.2.0188

4. Castellanos M, Leira R, Tejada J, Gil-Peralta A, Davalos A, et al. Predictors of good outcome in medium to large spontaneous supratentorial intracerebral haemorrhages. $J$ Neurol Neurosurg Psychiatry. 2005;76:691-695. https://doi.org/ 10.1136/jnnp.2004.044347

5. Castillo J, Davalos A, Alvarez-Sabin J, Pumar $\mathrm{JM}$, Leira R, et al. Molecular signatures of brain injury after intracerebral hemorrhage. Neurology. 2002;58:624-629. https://doi.org/10.1212/WNL .58 .4 .624

6. Di Napoli M, Papa F, Bocola V. C-reactive protein in ischemic stroke: an independent prognostic factor. Stroke. 2001;32:917-924. https://doi. org/10.1161/o1.STR.32.4.917

7. Di Napoli M, Papa F, Bocola V. Prognostic influence of increased C-reactive protein and fibrinogen levels in ischemic stroke. Stroke 2001;32:133-138. https://doi.org/10.1161/o1. STR.32.1.133

8. Di Napoli M, Schwaninger M, Cappelli R, Ceccarelli E, Di Gianfilippo G, Donati C, et al. Evaluation of C-reactive protein measurement for assessing the risk and prognosis in ischemic stroke: a statement for health care professionals from the CRP Pooling Project members. Stroke. 2005;36:1316-1329. https://doi.org/10.1161/o1. STR.0000165929.78756.ed

9. Dziedzic T: Clinical significance of acute phase reaction in stroke patients. Front Biosci. 2008;13:2922-2927. https://doi. org/10.2741/2897

10. Franke CL, van Swieten JC, Algra A, et al. Prognostic factors in patients with intracerebral haematoma. J Neurol Neurosrg Psychiatry. 1992;55:653-657. https://doi.org/10.1136/jnnp. 55.8 .653

11. Godoy DA, Papa F, Campi V, del Valle M, Piñero $\mathrm{G}$, et al. Relationship between baseline white blood cell and c-reactive protein with mortality in patients with spontaneous intracerebral hemorrhage. J Neurol Neurophysiol. 2010;1:104. https://doi.org/10.4172/2155-9562.1000104
12. Gong C, Hoff JT, Keep RF. Acute inflammatory reaction following experimental intracerebral hemorrhage in rat. Brain Res. 2000;871:57-65. https://doi.org/10.1016/Sooo6-8993(00)024276

13. Hanel RA, Xavier AR, Muhammad Y, Kirmani JF et al.: Outcome following intracerebral haemorrhage and subarachnoid haemorrhage. Neurol Res. 2002;24:58-62. https://doi. org/10.1179/016164102101200041

14. Karnik R, Valentin A, Ammerer HP, Hochfelner A, Donath $\mathrm{P}$, et al. Outcome in patients with intracerebral haemorrhage: predictors of survival. Wien Klin Wochenschr. 2000;112:169-173.

15. Keep RF, Xi G, Hua Y, Hoff JT. The deleterious or beneficial effects of different agents in intracerebral hemorrhage: think big, think small, or is hematoma size important? Stroke. 2005;36:1594-1596. https://doi.org/10.1161/o1. STR.0000170701.41507.e1

16. Kothari RU, Brott T, Broderick JP, Barsan WG, Sauerbeck LR, et al. The ABCs of measuring intracerebral hemorrhage volumes. Stroke. 1996;27:1304-1305. https://doi.org/10.1161/o1. STR.27.8.1304

17. Leira R, Davalos A, Silva Y, Gil-Peralta A, Tejada J. Early neurologic deterioration in intracerebral hemorrhage: predictors and associated factors. Neurology. 2004;63:461-467. https://doi. org/10.1212/01.WNL.0000133204.81153.AC

18. Loftspring MC, Hansen C, Clark JF. A novel brain injury mechanism after intracerebral hemorrhage: the interaction between heme products and the immune system. Med Hypotheses. 2010;74(1):63-66. https://doi.org/10.1016/j. mehy.2009.08.002

19. Nilsson OG, Lindgren A, Brandt L, Saveland H. Prediction of death in patients with primary intracerebral hemorrhage: a prospective study of a defined population. $J$ Neurosurg. 2002;97:531536. https://doi.org/10.3171/jns.2002.97.3.0531

20. Pepys MB, Hirschfield GM. C-reactive protein: a critical update. $J$ Clin Invest. 2003;111:18051812. https://doi.org/10.1172/JCI200318921

21. Power C, Henry S, Del Bigio MR, Larsen PH, Corbett D, et al. Intracerebral hemorrhage induces macrophage activation and matrix metalloproteinases. Ann Neurol. 2003;53:731742. https://doi.org/10.1002/ana.10553 
22. Qureshi AI, Suri MF, Ling GS, Khan J, Guterman LR, et al. Absence of early proinflammatory cytokine expression in experimental intracerebral hemorrhage. Neurosurgery. 2001;49:416-420.

23. Roger VL, Go AS, Lloyd-Jones DM, Adams RJ. Heart disease and stroke statistics-2011 update: a report from the American Heart Association. Circulation. 2011;123(4):e18-e209. https://doi. org/10.1161/CIR.obo13e3182009701

24. Schwarz S, Hafner K, Aschoff A, Schwab S. Incidence and prognostic significance of fever following intracerebral haemorrhage. Neurology. 2000;54:354-361. https://doi.org/10.1212/WNL. 54.2.354

25. Silva Y, Leira R, Tejada J, Lainez JM, Castillo J, et al. Molecular signatures of vascular injury are associated with early growth of intracerebral hemorrhage. Stroke. 2005;36:86-91. https://doi. org/10.1161/o1.STR.0000149615.51204.ob

26. Smajlovic D, Salihovic D, Sinanovic O, et al. Analysis of risk factors, localization and 30 day prognosis of intracerebral haemorrhage. Bosn $J$ Basic Med. Sci. 2008;8(2):121-125.

27. Sun W, Peacock A, Becker J, Phillips-Bute B, Laskowitz DT, James ML. Correlation of leukocytosis with early neurological deterioration following supratentorial intracerebral hemorrhage. J Clin Neurosci. 2012;19:10961100. https://doi.org/10.1016/j.jocn.2011.11.020
28. Sung KC, Suh JY, Kim BS, Kang JH, Kim H, Lee MH, Park JR, and Kim SW: High sensitivity C-reactive protein as an independent risk factor for essential hypertension. Am J Hypertension. 2003;16:429-433. https://doi.org/10.1016/ So895-7061(03)00566-1

29. Van Asch CJ, Luitse MJ, Rinkel GJ, van der Tweel I, Algra A, Klijn CJ. Incidence, case fatality, and functional outcome of intracerebral haemorrhage over time, according to age, sex, and ethnic origin: a systematic review and meta-analysis. Lancet Neurology. 2010;9(2):167-176. https://doi. org/10.1016/S1474-4422(09)70340-0

30. Wang J. Preclinical and clinical research on inflammation after intracerebral hemorrhage. Prog Neurobiol. 2010;92:463-477. https://doi. org/10.1016/j.pneurobio.2010.08.001

31. Wang J, Dore S. Inflammation after intracerebral hemorrhage. $J$ Cereb Blood Flow Metab. 2007;27:894-908. https://doi.org/10.1038/ sj.jcbfm.9600403

32. Xi G, Keep RF, Hoff JT. Mechanisms of brain injury after intracerebral haemorrhage. Lancet Neurology. 2006;5:53-63. https://doi. org/10.1016/S1474-4422(05)70283-0 